\title{
Less Might Be More: Conduction Failure as a Factor Possibly Limiting the Efficacy of Higher Frequencies in rTMS Protocols
}

OPEN ACCESS

Edited by:

Gregor Thut,

University of Glasgow,

United Kingdom

Reviewed by:

Veit Mylius,

Philipps University of Marburg,

Germany

Robin Cash,

Monash Alfred Psychiatry Research

Centre, Australia

*Correspondence:

Islam Halawa

islam.halawa@med.uni-goettingen.de

Specialty section:

This article was submitted to

Perception Science,

a section of the journal

Frontiers in Neuroscience

Received: 03 November 2017

Accepted: 08 May 2018

Published: 28 May 2018

Citation:

Halawa I, Goldental A, Shirota Y

Kanter I and Paulus W (2018) Less Might Be More: Conduction Failure as a Factor Possibly Limiting the Efficacy

of Higher Frequencies in rTMS

Protocols. Front. Neurosci. 12:358.

doi: 10.3389/fnins.2018.00358

\author{
Islam Halawa ${ }^{1 *}$, Amir Goldental $^{2}$, Yuichiro Shirota ${ }^{1}$, Ido Kanter ${ }^{2,3}$ and Walter Paulus ${ }^{1}$ \\ ' Department of Clinical Neurophysiology, University Medical Center Göttingen, Göttingen, Germany, ${ }^{2}$ Department of \\ Physics, Bar-llan University, Ramat-Gan, Israel, ${ }^{3}$ Goodman Faculty of Life Sciences, Gonda Interdisciplinary Brain Research \\ Center, Bar-llan University, Ramat-Gan, Israel
}

Introduction: rTMS has been proven effective in the treatment of neuropsychiatric conditions, with class A (definite efficacy) evidence for treatment of depression and pain (Lefaucheur et al., 2014). The efficacy in stimulation protocols is, however, quite heterogeneous. Saturation of neuronal firing by HFrTMS without allowing time for recovery may lead to neuronal response failures (NRFs) that compromise the efficacy of stimulation with higher frequencies.

Objectives: To examine the efficacy of different rTMS temporal stimulation patterns focusing on a possible upper stimulation limit related to response failures. Protocol patterns were derived from published clinical studies on therapeutic rTMS for depression and pain. They were compared with conduction failures in cell cultures.

Methodology: From 57 papers using protocols rated class A for depression and pain (Lefaucheur et al., 2014) we extracted Inter-train interval (ITI), average frequency, total duration and total number of pulses and plotted them against the percent improvement on the outcome scale. Specifically, we compared $10 \mathrm{~Hz}$ trains with ITIs of $8 \mathrm{~s}$ (protocol A) and $26 \mathrm{~s}$ (protocol B) in vitro on cultured cortical neurons.

Results: In the in vitro experiments, protocol A with 8-s ITIs resulted in more frequent response failures, while practically no response failures occurred with protocol B (26-s intervals). The HFrTMS protocol analysis exhibited no significant effect of ITIs on protocol efficiency.

Discussion: In the neuronal culture, longer ITIs appeared to allow the neuronal response to recover. In the available human dataset on both depression and chronic pain, data concerning shorter ITIs is does not allow a significant conclusion.

Significance: NRF may interfere with the efficacy of rTMS stimulation protocols when the average stimulation frequency is too high, proposing ITIs as a variable in rTMS protocol efficacy. Clinical trials are necessary to examine effect of shorter ITIs on the clinical outcome in a controlled setting.

Keywords: HfrTMS, rTMS, ITIs, NRFs, neuronal cultures 


\section{INTRODUCTION}

Repetitive transcranial magnetic stimulation (rTMS) is a noninvasive therapeutic tool for a variety of neuropsychiatric conditions (Lefaucheur et al., 2014). There are presently 617 ongoing clinical trials registered at https://clinicaltrials.gov/ (accessed 05.03.2018). There is class A evidence of the therapeutic utility of rTMS in the treatment of depression and chronic pain (Lefaucheur et al., 2014) which led the FDA to approve the treatment in the USA and Canada for depression (Lefaucheur et al., 2014).

On the other hand, rTMS used in the treatment of other disorders, such as panic disorders, hallucinations, obsessivecompulsive disorder, schizophrenia, Parkinson's disease, dystonia and stroke has been less promising so far (Lefaucheur et al., 2014). To increase the use of rTMS and its acceptance in the medical community across different medical specialties requires a better understanding of the potential pitfalls of the employed protocols.

As far as stimulation frequency is concerned, there is some consensus on the excitatory effects at higher frequencies and inhibitory effects at lower frequencies (Fitzgerald et al., 2006), although further temporal variants also play a role.

However, the temporal organization of rTMS pulses, including the inter-train interval (ITI) or intra-burst interval, has attracted less attention, despite its seemingly cardinal role in determining rTMS efficacy. The relevance of the ITI has been more intensively studied in so-called "patterned stimulation protocols" such as theta burst (Huang et al., 2005) or quadripulse (Hamada et al., 2008) stimulation. In these paradigms, a smaller number of stimulations was more efficacious than rTMS with a constant inter-stimulation interval. However, the influence of the ITI needs to be better addressed in the conventional stimulation protocols used in the treatment of depression or chronic pain.

Different time ranges play different roles in this context. In TBS it seems that the introduction of an 8-s ITI between ten bursts of three high-frequency pulses at $5 \mathrm{~Hz}$ (i.e., in a theta range) was facilitatory, while cTBS without this 8-s ITI was inhibitory (Huang et al., 2005). With QPS the effect on plasticity induction is quite the opposite when intra-burst intervals within the burst of four are crossed over from facilitation when using $5 \mathrm{~ms}$ to inhibition when using $50 \mathrm{~ms}$ (Hamada et al., 2008). Even for conventional $5 \mathrm{~Hz}$ rTMS protocols the introduction of a longer ITI switched the aftereffects from inhibition to excitation (Rothkegel et al., 2010).

Another way to view the issue of ITI is to apply information gained from paired-pulse TMS protocols. For example, repetitive paired-pulse stimulation using $10-15 \mathrm{~ms}$ intervals, which can cause facilitation in the intracortical facilitation (ICF) protocol, was more excitatory than $2-3 \mathrm{~ms}$ intervals, which would cause inhibition in the short-interval intracortical inhibition (SICI) (Sommer et al., 2001; Shirota et al., 2016). In this way, even though ITIs were initially introduced for practical reasons, such as to avoid coil overheating or for probably mistaken safety considerations to reduce the risk of seizures, it can be a critical parameter that determines the clinical efficacy of rTMS.

The problem is that infinite possibilities exist for manipulating intervals. For example, using $10 \mathrm{~ms}$ intervals in the short intra-cortical facilitation (ICF) range, repetitive paired-pulse stimulation was more excitatory than with $2 \mathrm{~ms}$ intervals both at 5 and $2 \mathrm{~Hz}$ repetition frequency (Sommer et al., 2001).

Other strategies for increasing efficacy are increasing the duration of the stimulation and concomitantly the total number of pulses, e.g., 54,000 stimuli over 3 days (George et al., 2014). But this strategy may fail: when the motor evoked potential is used as the biomarker with theta burst, prolonged, intermittent theta burst stimulation (1,200 pulses instead of 600) is inhibitory, i.e., efficacy not only declined, but the direction of the changes was even reversed when the number of stimulation pulses was doubled (Gamboa et al., 2010). tDCS also showed similar behavior with a reversal of aftereffects when two sessions were applied back-to-back compared to a single session (Monte-Silva et al., 2013).

Also the very simple parameter of rTMS frequency still remains insufficiently investigated. Frequencies higher than $10 \mathrm{~Hz}$ have rarely been used in conventional rTMS protocols. One reason for this being the increasing technical difficulties, in particular coil heating with higher frequencies or a possibly increased risk of seizures. The safety guidelines (Rossi et al., 2009 ) at $110 \%$ motor threshold assume a safe train duration of more than $5 \mathrm{~s}$ at $10 \mathrm{~Hz}$ that decreases to $1.6 \mathrm{~s}$ at $15 \mathrm{~Hz}$ and $0.84 \mathrm{~s}$ at $25 \mathrm{~Hz}$.

\section{Neuronal Response Failures: From Cell Cultures to Human Brains}

Given the uncertainty of the significance of the temporal organizations in rTMS, we hypothesized that delayed neuronal response latency (NRL) or a resulting neuronal response failure (NRF) should play an important role in the biological effects of rTMS. When a neuron is subjected to supra-threshold stimulation, it typically produces an action potential, which can be measured extracellularly several milliseconds after the stimulation. The time-gap between the stimulation and the corresponding recorded evoked spike is known as neuronal response latency (Vardi et al., 2012; Goldental, 2014; Sardi et al., 2017, 2018). If the stimulus frequency is low enough, NRL is stable and there are no response failures. With repeated stimulations above a so-called critical frequency $\left(f_{c}\right)$, the NRL was found to stretch gradually (Figure 1A) (Goldental et al., 2016) until it fluctuated around an average value, and stochastic neuronal response failures (NRFs) appeared, i.e., the average firing rate is saturated and is equal to $f_{c}$, even if the external stimulation frequency is higher. The neuron functions like a lowpass filter. At a stimulation frequency higher than $f_{c}$ NRFs appear randomly and independently with a probability of $\mathrm{f}_{\mathrm{c}} / \mathrm{f}$ (Goldental et al., 2015). After several minutes without stimulation, the NRL approaches its initial value (Vardi et al., 2012). On the other hand, when a neuron is stimulated below its $\mathrm{f}_{\mathrm{c}}$, the NRL is stable (Vardi et al., 2015), and the probability of neuronal response failure is negligible (Vardi et al., 2015).

\section{Repetitive Transcranial Magnetic Stimulation and Neuronal Plasticity}

Thus, at the single neuron level, the response probability decreases when the stimulation frequency exceeds $f_{c}$ (Figure 1B; 

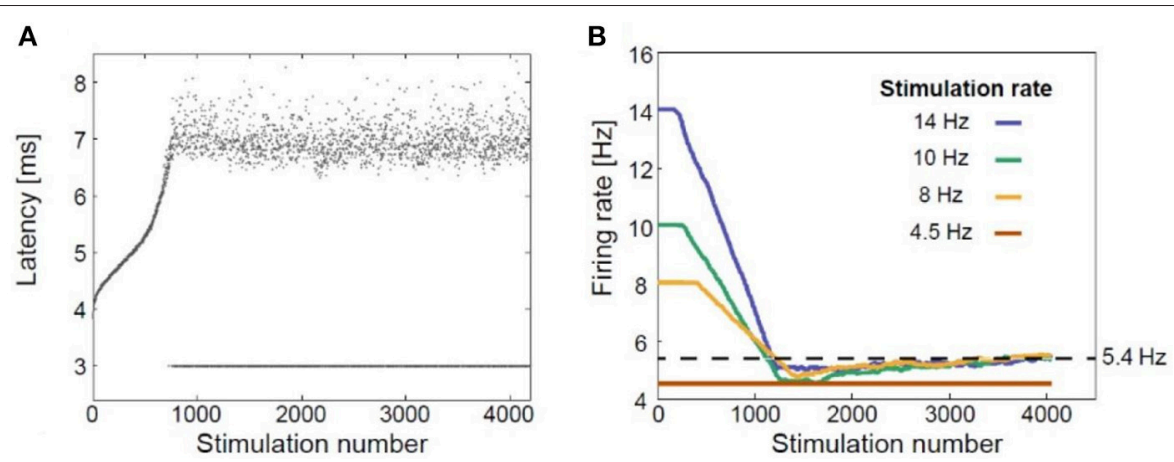

FIGURE 1 | Neuronal plasticity. (A) The neuronal response latency (NRL) of a cultured cortical neuron functionally separated from its network using a pharmacologic block, stimulated at a frequency of $10 \mathrm{~Hz}$. Response failures are represented by dots at $\mathrm{NRL}=3 \mathrm{~ms}$. (B) Firing rates for different stimulation rates using a sliding window of 1,000 stimulations, indicating a saturated firing rate $(\sim 5.4 \mathrm{~Hz}$, dashed line) independent of the applied stimulation rate. Adapted from Goldental et al. (2016) with the author's permission.

Goldental et al., 2016). At the population level, e.g., in recurrent or feed-forward networks, this effect is even enhanced because a sufficient number of neurons must fire in synchrony for the signal to propagate among populations of neurons representing perceptual entities (Vardi et al., 2012).

As a result, on the one hand, we expect rTMS with a lower frequency to be more reliable and thus more efficient than higher frequency stimulation, in which neuronal response lead to less synchronized responses. On the other hand, we must differentiate between intended inhibitory effects that occur with rTMS frequencies around $1 \mathrm{~Hz}$, and rTMS stimulation with frequencies higher than $1 \mathrm{~Hz}$, which are excitatory. The frequency/response curve should therefore resemble an inverted $\mathrm{U}$. The situation is however more complicated when considering intervals, which allow a recovery of conduction reliability as outlined in more detail in the comparison of two trials on rTMS in depression.

The influence of the inter-train interval in rTMS has been investigated less than that of stimulation frequency (e.g., 1 or $5 \mathrm{~Hz}$ ) even in the context of basic research, so we examined the premise using the neuronal culture developed by Vardi and coworkers (Vardi et al., 2015). The greatest incremental success in the treatment of depression was seen in a key trial (O'Reardon et al., 2007) using a special patterned paradigm based on $10 \mathrm{~Hz}$ rTMS, while the results of a different multicenter trial conducted at the same time using $10 \mathrm{~Hz}$ rTMS in the treatment of refractory depression were negative (Herwig et al., 2007). Among the many reasons for the contrary outcomes, e.g., total stimulation duration, number of stimuli per session, total duration in days and intensity, the two studies differed in the pattern of their rTMS stimulation sequence. The successful data set (Protocol B) applied 3,000 $10 \mathrm{~Hz}$ stimuli per day with $4 \mathrm{~s}$ of stimulation and an interval of $26 \mathrm{~s}$. The unsuccessful study (Protocol A) applied $2,00010 \mathrm{~Hz}$ stimuli per day with $2 \mathrm{~s}$ of stimulation and an 8-s interval without stimulations. It should be noted that the Herwig Protocol (Herwig et al., 2007) used an accelerated treatment protocol of 15 days, while the O'Reardon Protocol (O'Reardon et al., 2007) used a full course of 4-6 weeks of treatment.
Treatment duration is also known to be a factor that influences treatment efficacy (Lefaucheur et al., 2014). Nonetheless, ITI duration is also an important parameter consideration, which may influence the efficacy of clinical protocols.

We compared both protocols using a single neuron in vitro approach in a cell culture model (Goldental et al., 2016).

In summary, we investigated in a literature review whether conduction failure of cortical neurons limited reliable spike conduction by comparing the published results of clinical studies with in vitro experiments.

\section{METHODS}

Protocols A and B were compared in a cell culture (Vardi et al., 2012; Sardi et al., 2017). All cell culture experimental procedures were conducted in accordance with the National Institutes of Health Guide for the Care and Use of Laboratory Animals and Bar-Ilan University Guidelines for the Use and Care of Laboratory Animals in Research and are approved and supervised by the Institutional Animal Care and Use Committee. Cortical neurons are obtained from newborn rats within $48 \mathrm{~h}$ after birth using mechanical and enzymatic procedures (Vardi et al., 2012; Sardi et al., 2017). The neurons are plated directly onto substrate-integrated multi-electrode arrays (MEAs) and are allowed to develop functionally and structurally mature networks over a period of 2-3 weeks in-vitro, prior to the experiments. The number of plated neurons in a typical network is in the order of 1,300,000, covering an area of about $380 \mathrm{~mm}^{2}$. In order to conduct experiments in which cultured cortical neurons are functionally isolated from their network, a pharmacological block of glutamatergic and GABAergic synapses is performed. This cocktail does not block the spontaneous network activity completely, but rather makes it sparse. At least $1 \mathrm{~h}$ is allowed for stabilization of the effect. For stimulation and recording an array of 60 extracellular electrodes, $30 \mu \mathrm{m}$ in diameter, and spaced 200$500 \mu \mathrm{m}$ from each other (Multi-Channel Systems, Reutlingen, Germany) is used. Mono-phasic square voltage pulses were used, in the range of $[-800,-500] \mathrm{mV}$ and $[60,400] \mu \mathrm{s}$ and each 
channel was sampled at a frequency of $50 \mathrm{k}$ samples/s (Vardi et al., 2015). Post-experiment analyses are performed in a Matlab environment (MathWorks, Natwick, MA, USA).

For a more realistic setting, considering that neurons are not at rest before rTMS, they were primed with 300 stimulations at $10 \mathrm{~Hz}$ followed by 30 repetitions of the stimulation patterns according to the protocols A or B (Figure 2).

For the simulation, a critical frequency $\left(f_{c}\right)$ was randomly chosen from range of 1 to $10 \mathrm{~Hz}$ frequency for each neuron. The neuronal response latency was simulated as a constant increase per firing, $0.03 \mathrm{~ms}$, for stimulation frequencies above the neuronal $f_{c}$ and an exponential decay with a time constant of $40 \mathrm{~s}$ for lower frequencies. Above the critical neuronal response latency increase of $3 \mathrm{~ms}$ neuronal response failures occur and the NRL is set as a random value between 3 and $4 \mathrm{~ms}$ for each firing of the neuron. The connections between layers in the feed-forward networks were randomly chosen with a probability of $15 / \mathrm{N}$ and a strength equal to 0.1 of the neuronal threshold, where $\mathrm{N}$ is the number of neurons in a layer. All other details are the same as in Vardi et al. (2015).

We simulated protocol A neurons in layer 1 with suprathreshold stimulations at $10 \mathrm{~Hz}$ for $2 \mathrm{~s}$, every $10 \mathrm{~s}$, and for protocol B we stimulated neurons in layer 1 with supra-threshold stimulations at $10 \mathrm{~Hz}$ for $4 \mathrm{~s}$, every $30 \mathrm{~s}$.

To verify the ITI-influence hypothesis from available data on depression studies, we obtained 59 detailed protocols from 57 papers with class A level of evidence. We also focused on the number of pulses per train, number and duration of trains, interval duration, and number of sessions (Tables 1, 2). Forty of the protocols were for depression, with two studies (George et al., 2000; Su et al., 2005) directly comparing two protocols, and 19 were for the treatment of pain as summarized in Lefaucheur et al. (2014). High frequency rTMS stimulation was applied to the left DLPFC for depression, and to the contralateral motor area for pain (Lefaucheur et al., 2014).
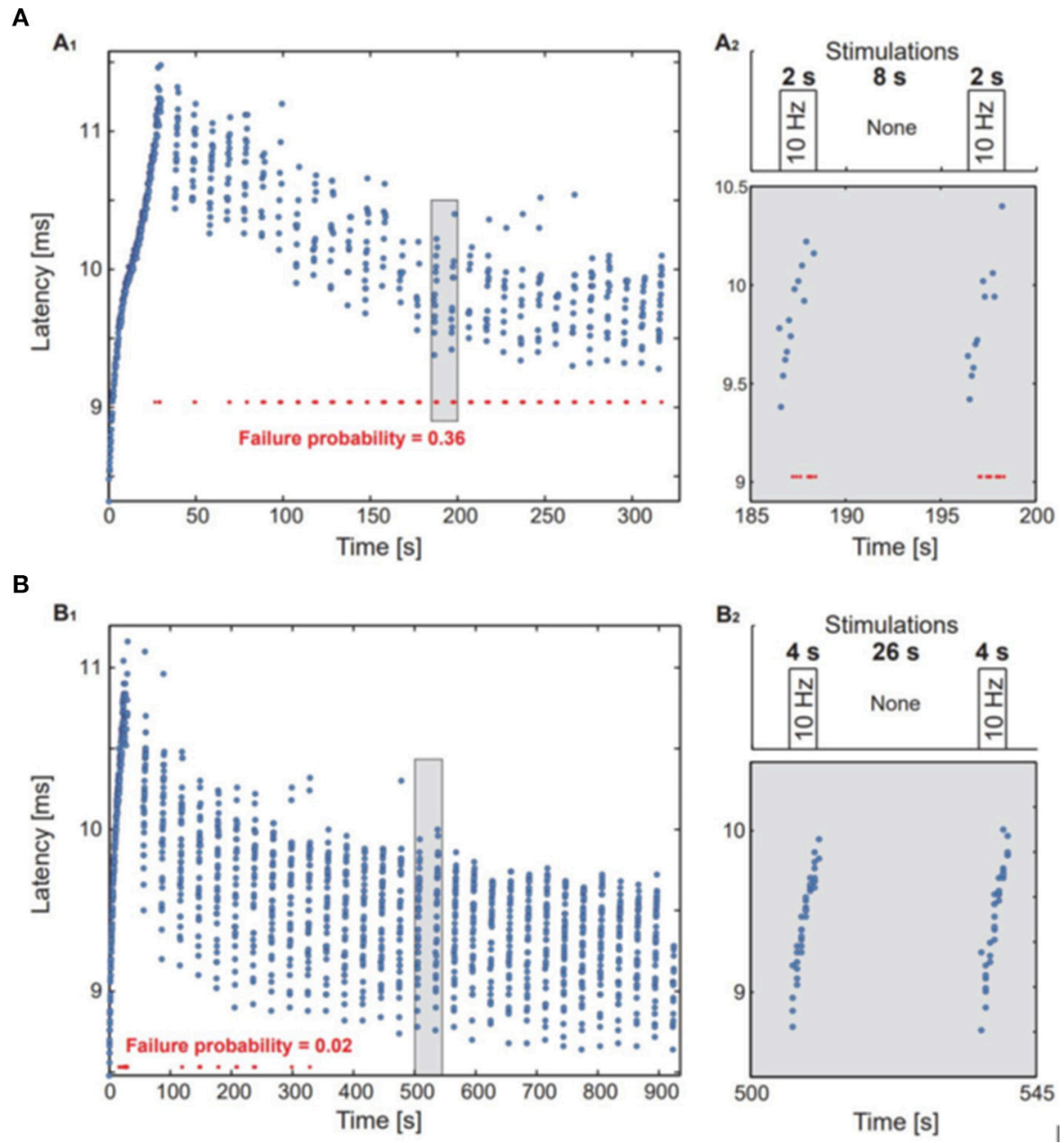

FIGURE 2 | In vitro experiments in single neurons. A neuron with an $\mathrm{fC}=1.3 \mathrm{~Hz}$ was stimulated above the threshold using the stimulation timings of protocols $\mathrm{A}$ and B. (A1,B1) The neuronal response latency was measured for each response, response failures are represented by red dots. (A2,B2) upper panel: an illustration of the stimulation protocol. Lower panel: a zoom-in of the marked area at (A1,B1). 


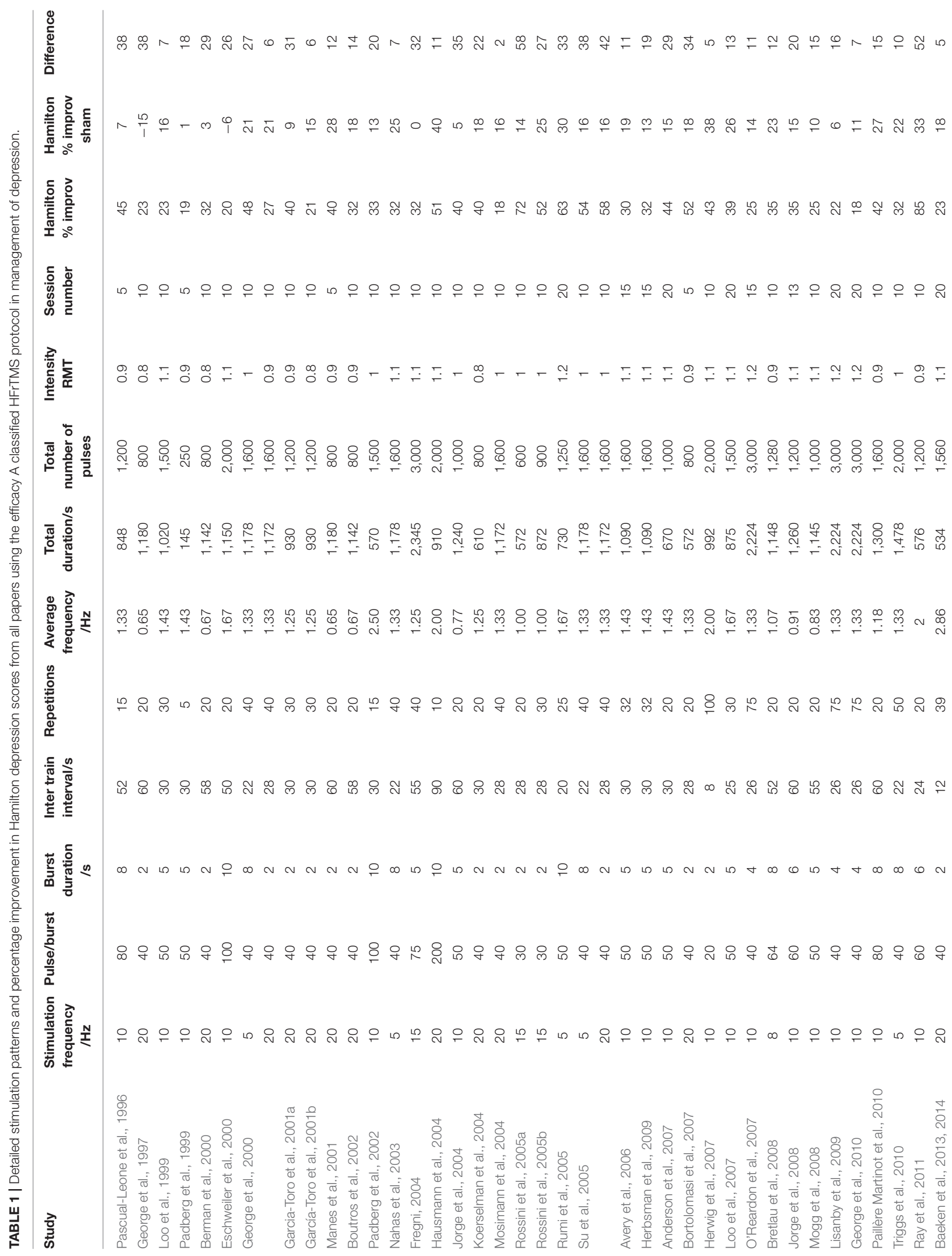




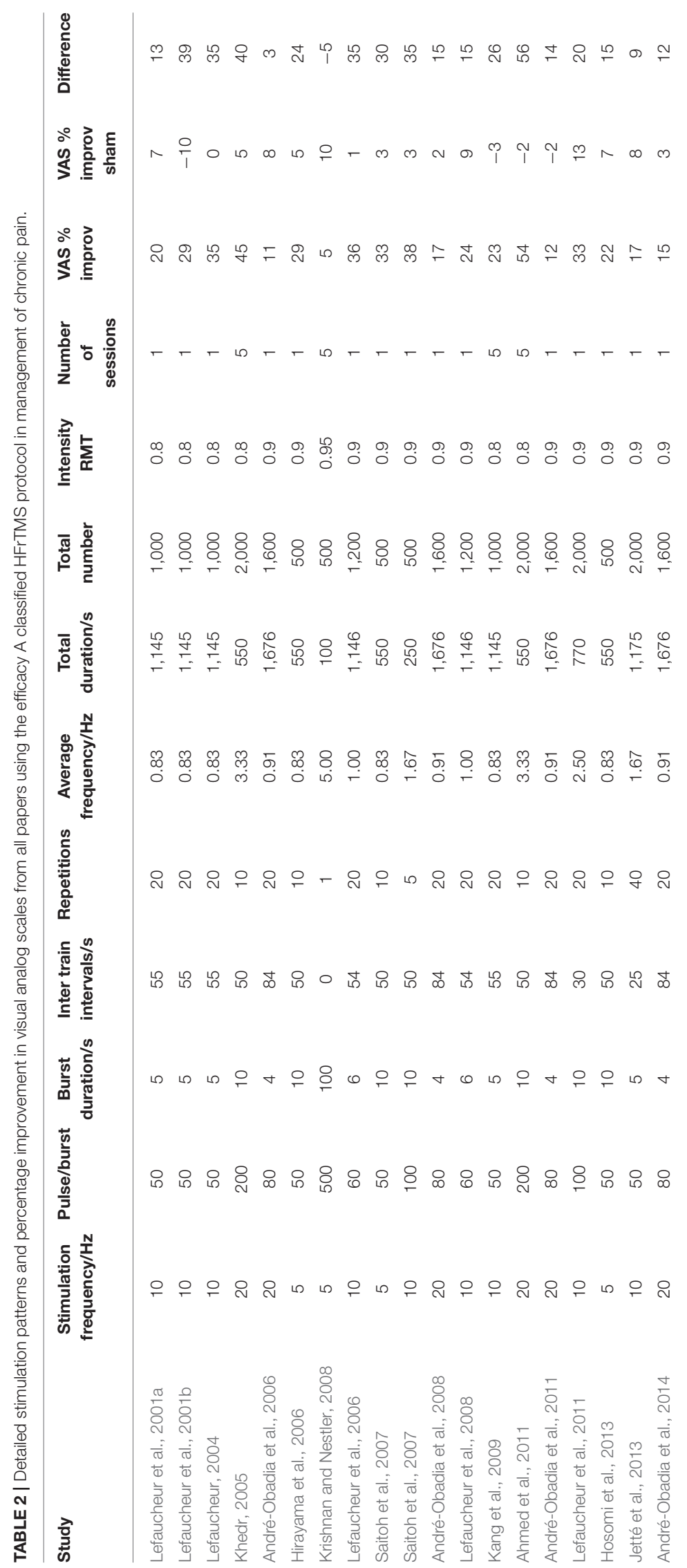


The parameters studied were average stimulation frequency and total duration of stimulation. In the published depression studies, we assessed efficacy as the percentage improvement in the Hamilton depression rating scale (HDRS) compared to sham conditions. In the studies on chronic pain, we assessed changes in pain severity quantified with a visual analog scale (VAS). We plotted the results against average stimulation frequency, interval duration, total duration and total number of pulses. We excluded the study by Fregni (2004) from the analysis because it did not compare rTMS to sham stimulation but only to drug therapy with both showing an equal degree of improvement.

\section{RESULTS}

In the cell culture study, protocol A with the shorter ITIs was associated with a substantial fraction of response failures, while these were much less frequent in protocol B (Figure 3). The average stimulation frequency in protocol A was $2 \mathrm{~Hz}(20$ stimulations per $10 \mathrm{~s}$ ) as compared to the average stimulation frequency of $1.33 \mathrm{~Hz}$ in protocol B (40 stimulations per $30 \mathrm{~s}$ ).

Figure 3 illustrates, through simulations, the superiority of protocol B over protocol A for both single neurons and networks, i.e., higher response probability when applying protocol B. Simulations of feedforward, layered networks increasingly demonstrated this effect, which was also visible in simulations including more than two layers (Figure 4).

\section{HFrTMS Protocol Evaluation}

Our evaluation of the available clinical data in a literature review showed that ITI protocols using ITIs of $20 \mathrm{~s}$ and longer were superior to shorter ITI protocols in the treatment of depression and chronic pain. For depression, the relationship between ITIs and improvement in HDRS was not linear and followed an inverted U-curve. We must, however, emphasize that only two studies used ITIs shorter than $20 \mathrm{~s}$ in the treatment of depression, and that there was only one such protocol for chronic pain.

We plotted the efficacy against isolated parameters, clustering the data into four groups and connecting the centers of the clusters. The graphs, with the exception of Figure 6A, showed low coefficients of determination $\left(R^{2}\right)$, which suggests poor fitting of the graph to the data points. Only the plot of ITIs against VAS improvement in Figure 6A showed an $R^{2}$ of 0.53 and a $P$ of 0.019 .

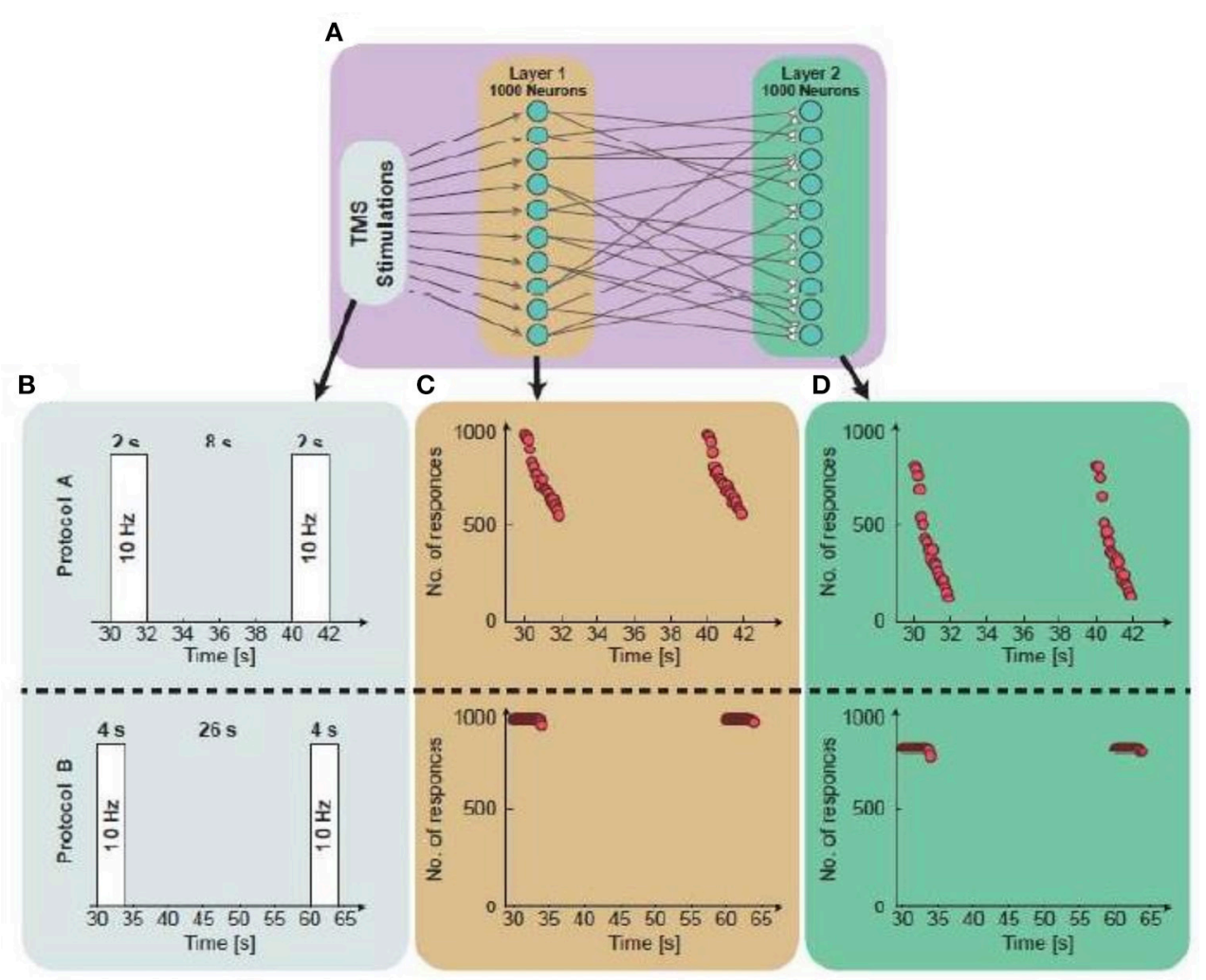

FIGURE 3 | Simulations from a single neuron to population dynamics. (A) A scheme of the simulated two-layered feed-forward network: Two populations, 1st layer is randomly connected presynaptically to the 2 nd layer. Each layer consists of 1,000 neurons and the 1st layer is stimulated repeatedly. (B) An illustration of the simulated protocol. Upper panel: Neurons in the 1st layer are stimulated at $10 \mathrm{~Hz}$ for $2 \mathrm{~s}$, every $10 \mathrm{~s}$. Lower panel: Neurons in the $1 \mathrm{st}$ layer are stimulated at $10 \mathrm{~Hz}$ for $4 \mathrm{~s}$, every $30 \mathrm{~s}$. (C) The number of firing neurons in the 1 st layer as a function of the stimulation time. The average number of responses in the $1 \mathrm{st}$ layer for protocol A and protocol B is 0.75 and 0.999 , respectively. (D) The number of firing neurons in the 2nd layer as a function of the stimulation time. The response probability in the 2nd layer for protocols $A$ and $B$ is 0.44 and 0.85 , respectively. 


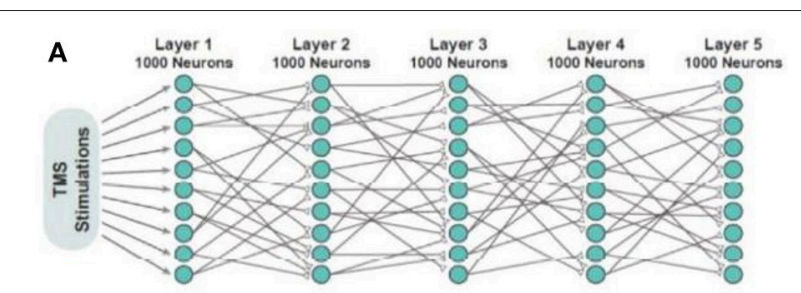

B

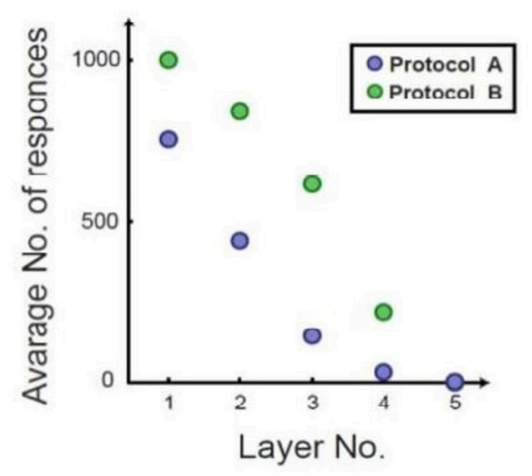

FIGURE 4 | Simulation of a feed-forward network consisting of five layers. (A) A diagram of the simulated feed-forward network. (B) The average number of responses as a function of the layer number. In the presented case, protocol B demonstrated fewer response failures in comparison to protocol $\mathrm{A}$ up to the 5th layer.

Plotting the inter-train intervals against effect showed that the most effective duration between $10 \mathrm{~Hz}$ trains was approximately $50 \mathrm{~s}\left(R^{2}=0.147\right.$; Figure 5A).

Moreover, the average frequency in the sessions (calculated by dividing the total number of pulses by the total session time in seconds) showed a negative correlation with the efficacy measure, i.e., the higher the average frequency, the less effective the treatment (Figure 5B). As mentioned above, protocol B employed a lower average frequency than protocol A $\left(R^{2}=0.143\right)$.

The evaluated studies on chronic pain revealed similar tendencies (Figures 6A,B) with an optimum inter-train interval also around $50 \mathrm{~s}\left(R^{2}=0.53\right)$ and an average frequency of about $2.5 \mathrm{~Hz}\left(R^{2}=0.36\right)$.

Both total duration of stimulation and the number of pulses showed a negative correlation with efficiency in depression studies. Protocols lasting more than $20 \mathrm{~min}$ were less effective than shorter ones (Figure 5C) $(P$-value $=0.041)$. Protocols using more than 2,000 pulses had a lower efficacy than protocols with a smaller number of pulses (Figure 5D) $(P$-value $=0.113$ using independent samples $t$-test $)$. Thus, it seems that the average frequency is a more important measure than simply the number of pulses. For example, in our comparison protocol B employed 3,000 pulses lasting approximately $37 \mathrm{~min}$ while protocol A, used 2,000 pulses over $16.5 \mathrm{~min}$.

This was also evident in the pain studies (Figure 6C), which showed a decrease in efficiency with longer stimulation $\left(R^{2}=\right.$ 0.33 ). No correlation was found with the total number of pulses (Figure 6D).

\section{DISCUSSION}

We evaluated the hypothesis that an increase in the effect magnitude of rTMS cannot be achieved by deliberately increasing the number of TMS pulses per time unit because of the provocation of conduction failures. We compared the results of published evidence level A studies in pain and depression with data obtained in cell cultures. In chronic pain protocols we found an inverse relationship between excitatory rTMS frequency and efficacy as predicted, but we were unable to verify this for the depression protocols. We believe that this is because of the differences in the neuronal circuitries involved in the two disorders, with different optimum firing and stimulation frequencies (Krishnan and Nestler, 2008; Simons et al., 2014).

The analysis of the depression studies revealed that most research groups used more or less similar protocols leading to the data being clustered around certain points. Other significant points, e.g., ITIs of ca. $50 \mathrm{~s}$ and an average frequency of ca. $1.5 \mathrm{~Hz}$ are lacking and should be tested. Despite the fact that relevant points are missing and that the data is noisy, we were still able to detect a pattern in the graphical representations, in which the missing testable variables are easily visible.

In the studies on chronic pain, that of Irlbacher et al. (2006) seems to stand out and illustrates the importance of the ITIs. They used a 100-s continuous train of $5 \mathrm{~Hz}$ rTMS (hence the short duration and small number of pulses) without any intervals. Of all the pain studies reviewed here this is the only one that actually showed a worsening of visual analog scales scores compared to sham stimulation. This supports the results of an earlier study that described inhibition using $5 \mathrm{~Hz}$ rTMS without intervals (Rothkegel et al., 2010). In all other pain studies the number of pulses per burst ranged from 50 to 200 (for $20 \mathrm{~Hz}$ ).

We are aware that conduction failures play only one, and probably a minor role compared to the multitude of other plasticity mechanisms. The pleomorphism in response can be mediated by the effect of such patterns and parameters on the behavior of intracellular calcium (Huang et al., 2011; Wilson et al., 2016). Glutamatergic transmission, in particular NMDA receptor mechanisms also play key roles.

A low-pass filter effect of single neurons and, consequently, of neuronal networks is demonstrated by the observation that when a single neuron is stimulated at frequencies of between 20 and $100 \mathrm{~Hz}$, its actual maximum firing frequency is capped at, for example, $17 \mathrm{~Hz}$ (Sardi et al., 2016). Even at a continuous $10 \mathrm{~Hz}$ stimulation frequency, the neuronal response latencies increase with significant response failures occurring after 700 stimulations.

Neuronal cell culture results showed protocol B to be more efficient than protocol $\mathrm{A}$, with the difference attributed to the longer silence period of $26 \mathrm{~s}$ used in protocol B that allowed recovery and relaxation of the NRL toward its initial value. i.e., the higher average stimulation frequency in protocol $\mathrm{A}$ is interpreted as a main source of the difference between their efficiencies.

A second finding derived from the cell culture data was that firing was initially stable but that conductions failures occurred after a few hundred stimuli. It is unclear whether this can be 

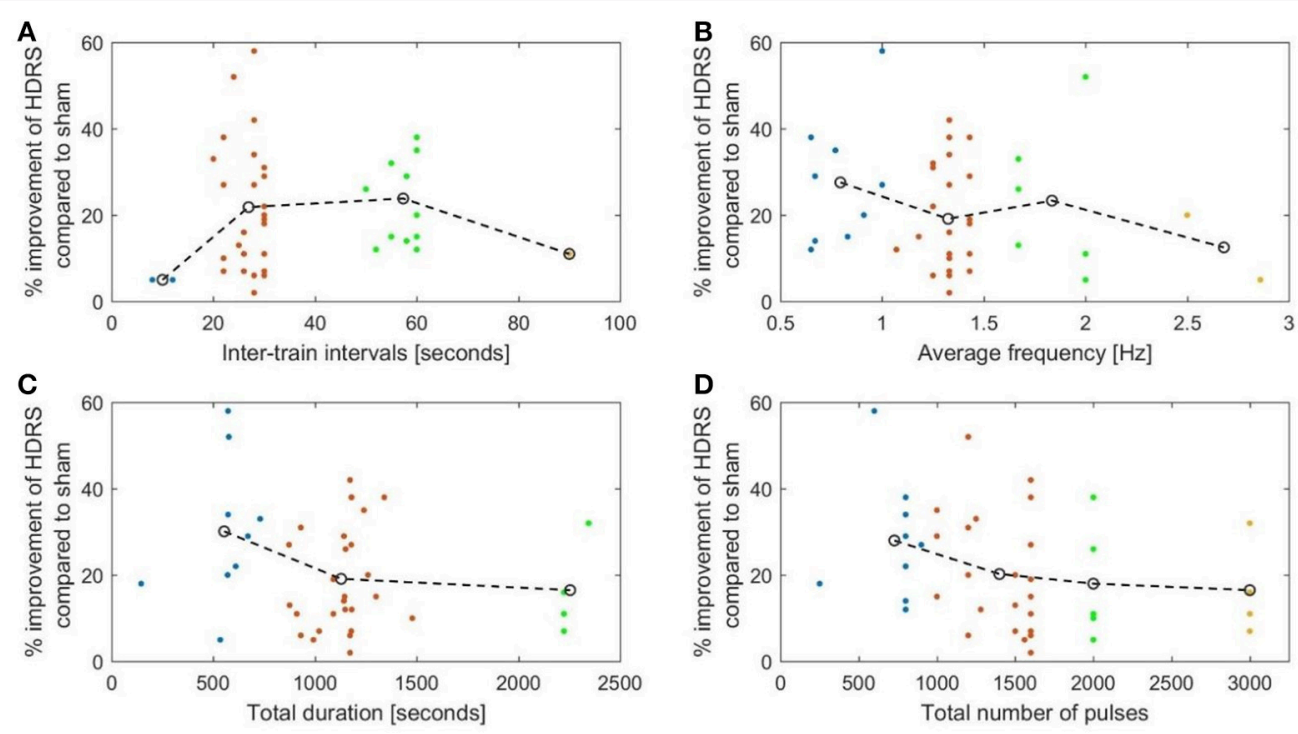

FIGURE 5 | The percentage reduction of the Hamilton depression rating score compared to sham plotted against. (A) The duration of the inter-stimulation intervals $\left(R^{2}=0.147\right)$. (B) The average stimulation frequency $\left(R^{2}=0.143\right)$. (C) The total duration of stimulation $\left(R^{2}=0.037\right)$. (D) The total number OF pulses $\left(R^{2}=0.113\right)$.
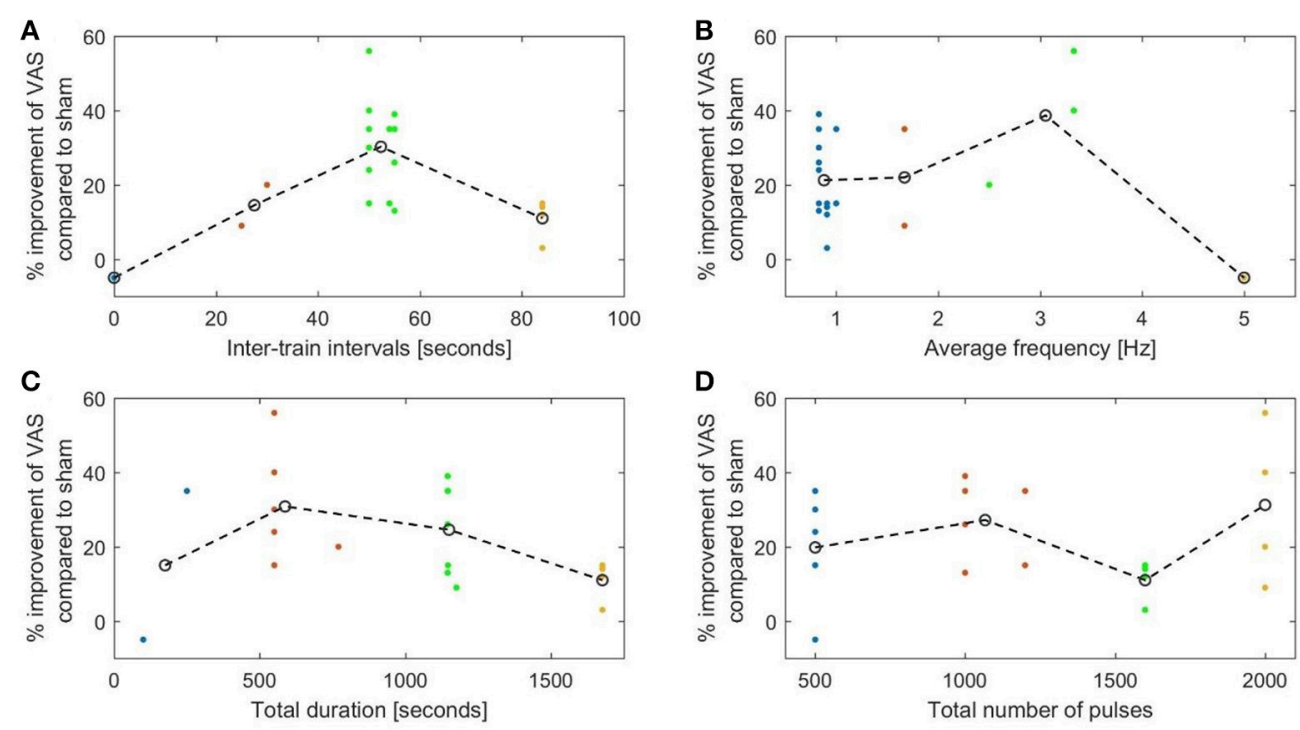

FIGURE 6 | The percentage decrease in VAS rated pain compared to sham plotted against. (A) The duration of the inter-stimulation intervals $\left(R^{2}=0.53\right)$. (B) The length of the average stimulation frequency $\left(R^{2}=0.36\right)$. (C) The total duration of stimulation $\left(R^{2}=0.33\right)$. (D) The total number of pulses $\left(R^{2}=0.24\right)$.

transferred to clinical studies, since no similar protocols have been used in patients.

Theoretically, the effect of response failures on the activity of a feed-forward network is enhanced in case of subthreshold synapses. While in the case of a single neuron, the neuron can fire or not-fire, in this case several neurons have to fire synchronously in order to transmit the signal to the next population.

A recent experiment investigated in normal subjects intertrain intervals ranging from 4 to $32 \mathrm{~s}$ for a $20 \mathrm{~Hz}$ protocol (Cash et al., 2017). At first glance the results seem to argue against our hypothesis; the shortest ITI using 4-s generated the highest MEPs, the $8 \mathrm{~s}$ intervals stimulation resulted in the smallest MEPs, which increased again with 16 and $32 \mathrm{~s}$ ITI. The 8 to $32 \mathrm{~s}$ ITI findings are however in line with our hypothesis. For the $4 \mathrm{~s}$ ITI finding a different mechanism may apply: Since SICI was disinhibited most by the shortest ITI of $4 \mathrm{~s}$ with a smaller disinhibition at $8 \mathrm{~s}$ and almost none at 16 and $32 \mathrm{~s}$. The large $4 \mathrm{~s}$ ITI disinhibition of SICI may overrun conduction failures and dominate the $4 \mathrm{~s}$ results in the MEP study (Cash et al., 2017). Almost certainly more than one mechanism is involved in the production of the net outcome. 
Controlled studies are also here necessary to split up the involved mechanisms.

Conduction failures are also determined by the original firing rate of the network so that the upper frequency limit might also apply to inhibitory protocols using $1 \mathrm{~Hz}$ stimulation frequencies. Repetition suppression (i.e., the decrease in the amplitude of subsequent MEPs compared to the first stimulus) is the measure to test for inhibitory protocols with lower frequencies (Pitkänen et al., 2017). With higher frequency facilitatory protocols using $5 \mathrm{~Hz}$, the MEP amplitudes increased during the train but inter-train suppression was also detected as a latent period stretching with each TMS pulse (Berardelli et al., 1999).

More data is required in order to optimize the protocol further. In future studies, one might consider starting stimulation with a higher rTMS pulse frequency incorporating a decay over time in order to remain below the critical frequency at which conduction blocks arise. rTMS protocols employing a higher frequency may be more efficient initially but become less reliable over time.

We conclude that depending on the disorder and the desired outcome it should be possible to optimize both the intervals between stimulation trains and the average stimulation frequencies.

\section{REFERENCES}

Ahmed, M. A., Mohamed, S. A., and Sayed, D. (2011). Long-term antalgic effects of repetitive transcranial magnetic stimulation of motor cortex and serum beta-endorphin in patients with phantom pain. Neurol. Res. 33, 953-8. doi: 10.1179/1743132811Y.0000000045

Anderson, I. M., Delvai, N. A., Ashim, B., Ashim, S., Lewin, C., Singh, V., et al. (2007). Adjunctive fast repetitive transcranial magnetic stimulation in depression. Br. J. Psychiatry 190, 533-4. doi: 10.1192/bjp.bp.106.028019

André-Obadia, N., Magnin, M., and Garcia-Larrea, L. (2011). On the importance of placebo timing in rTMS studies for pain relief. Pain 152, 1233-1237. doi: $10.1016 /$ j.pain.2010.12.027

André-Obadia, N., Mertens, P., Gueguen, A., Peyron, R., and GarciaLarrea, L. (2008). Pain relief by rTMS differential effect of current flow but no specific action on pain subtypes. Neurology 71, 833-840. doi: 10.1212/01.wnl.0000325481.61471.f0

André-Obadia, N., Mertens, P., Lelekov-Boissard, T., Afif, A., Magnin, M., and Garcia-Larrea, L. (2014). Is Life better after motor cortex stimulation for pain control? Results at long-term and their prediction by preoperative rTMS. Pain Physician 17, 53-62.

André-Obadia, N., Peyron, R., Mertens, P., Mauguière, F., Laurent, B., and Garcia-Larrea, L. (2006). Transcranial magnetic stimulation for pain control. Double-blind study of different frequencies against placebo, and correlation with motor cortex stimulation efficacy. Clin. Neurophysiol. 117, 1536-1544. doi: 10.1016/j.clinph.2006.03.025

Avery, D. H., Holtzheimer, P. E., Fawaz, W., Russo, J., Neumaier, J., Dunner, D. L., et al. (2006). A controlled study of repetitive transcranial magnetic stimulation in medication-resistant major depression. Biol. Psychiatry 59, 187-94. doi: 10.1016/j.biopsych.2005.07.003

Baeken, C., Marinazzo, D., Wu, G.-R., Van Schuerbeek, P., De Mey, J., Marchetti, I., et al. (2014). Accelerated HF-rTMS in treatment-resistant unipolar depression: insights from subgenual anterior cingulate functional connectivity. World J. Biol. Psychiatry 15, 286-297. doi: 10.3109/15622975.2013.872295

Baeken, C., Vanderhasselt, M.-A., Remue, J., Herremans, S., Vanderbruggen, N., Zeeuws, D., et al. (2013). Intensive HF-rTMS treatment in refractory
In the examined clinical data set for class A rTMS protocols, a lot of heterogeneity were observed in other stimulation variables such as stimulation frequencies, intensities, number of pulses and treatment duration, each one of which might solely explain the discrepancy between the clinical outcome of the O'Reardon et al. (2007) and Herwig et al. (2007) protocols. The focus of our work here was on ITIs, which were so far not considered as an isolated rTMS variable in all studies. Based on the neuronal culture results we propose ITIs as a noteworthy variable in therapeutic rTMS protocols. As such, it would be helpful to initiate specific studies in which only the ITI parameter is modified with a clinical outread.

\section{AUTHOR CONTRIBUTIONS}

IH gathered and analyzed data and wrote Manuscript. AG carried out the neuronal culture experiments, analyzed data and idea. YS gathered and analyzed data and revision. IK oversight and review. WP idea, oversight, and review.

\section{FUNDING}

The research of IK is supported in part by the TELEM grant of the Council for Higher Education of Israel.

medication-resistant unipolar depressed patients. J. Affect. Disord. 151, 625-631. doi: 10.1016/j.jad.2013.07.008

Berardelli, A., Inghilleri, M., Gilio, F., Romeo, S., Pedace, F., Currà, A., et al. (1999). Effects of repetitive cortical stimulation on the silent period evoked by magnetic stimulation. Exp. Brain Res. 125, 82-86. doi: 10.1007/s002210050661

Berman, R. M., Narasimhan, M., Sanacora, G., Miano, A. P., Hoffman, R. E., Hu, X. S., et al. (2000). A randomized clinical trial of repetitive transcranial magnetic stimulation in the treatment of major depression. Biol. Psychiatry 47, 332-337. doi: 10.1016/S0006-3223(99)00243-7

Bortolomasi, M., Minelli, A., Fuggetta, G., Perini, M., Comencini, S., Fiaschi, A., et al. (2007). Long-lasting effects of high frequency repetitive transcranial magnetic stimulation in major depressed patients. Psychiatry Res. 150, 181-186. doi: 10.1016/j.psychres.2006.04.010

Boutros, N. N., Gueorguieva, R., Hoffman, R. E., Oren, D. A., Feingold, A., and Berman, R. M. (2002). Lack of a therapeutic effect of a 2-week sub-threshold transcranial magnetic stimulation course for treatment-resistant depression. Psychiatry Res. 113, 245-254. doi: 10.1016/S0165-1781(02)00267-6

Bretlau, L., Lunde, M., Lindberg, L., Undén, M., Dissing, S., and Bech, P. (2008). Repetitive transcranial magnetic stimulation (rTMS) in combination with escitalopram in patients with treatment-resistant major depression. a double-blind, randomised, sham-controlled trial. Pharmacopsychiatry 41, 41-7. doi: 10.1055/s-2007-993210

Cash, R. F. H., Dar, A., Hui, J., De Ruiter, L., Baarbé, J., Fettes, P., et al. (2017). Influence of inter-train interval on the plastic effects of rTMS. Brain Stimul. 10, 630-636. doi: 10.1016/j.brs.2017.02.012

Eschweiler, G. W., Wegerer, C., Schlotter, W., Spandl, C., Stevens, A., Bartels, M., et al. (2000). Left prefrontal activation predicts therapeutic effects of repetitive transcranial magnetic stimulation (rTMS) in major depression. Psychiatry Res. Neuroimaging 99, 161-72. doi: 10.1016/S0925-4927(00)00062-7

Fitzgerald, P., Fountain, S., and Daskalakis, Z. (2006). A comprehensive review of the effects of rTMS on motor cortical excitability and inhibition. Clin. Neurophysiol. 117, 2584-2596. doi: 10.1016/j.clinph.2006.06.712

Fregni, F. (2004). Repetitive transcranial magnetic stimulation is as effective as fluoxetine in the treatment of depression in patients with Parkinson's disease. J. Neurol. Neurosurg. Psychiatry 75, 1171-1174. doi: 10.1136/jnnp.2003.027060 
Gamboa, O. L., Antal, A., Moliadze, V., and Paulus, W. (2010). Simply longer is not better: reversal of theta burst after-effect with prolonged stimulation. Exp. Brain Res. 204, 181-187. doi: 10.1007/s00221-010-2293-4

Garcia-Toro, M., Mayol, A., Arnillas, H., Capllonch, I., Ibarra, O., and Crespí, M., et al. (2001a). Modest adjunctive benefit with transcranial magnetic stimulation in medication-resistant depression. J. Affect. Disord. 64, 271-275. doi: 10.1016/S0165-0327(00)00223-8

García-Toro, M., Pascual-Leone, A., Romera, M., González, A., Micó, J., Ibarra, O., et al. (2001b). Prefrontal repetitive transcranial magnetic stimulation as add on treatment in depression. J. Neurol. Neurosurg. Psychiatry 71, 546-548. doi: 10.1136/jnnp.71.4.546

George, M. S., Lisanby, S. H., Avery, D., McDonald, W. M., Durkalski, V., Pavlicova, M., et al. (2010). Daily left prefrontal transcranial magnetic stimulation therapy for major depressive disorder: a shamcontrolled randomized trial. Arch. Gen. Psychiatry 67, 507-516. doi: 10.1001/archgenpsychiatry

George, M. S., Nahas, Z., Molloy, M., Speer, A. M., Oliver, N. C., Li, X.-B., et al. (2000). A controlled trial of daily left prefrontal cortex TMS for treating depression. Biol. Psychiatry 48, 962-970. doi: 10.1016/S0006-3223(00)01048-9

George, M. S., Raman, R., Benedek, D. M., Pelic, C. G., Grammer, G. G., Stokes, K. T., et al. (2014). A two-site pilot randomized 3 day trial of high dose left prefrontal repetitive transcranial magnetic stimulation (rTMS) for suicidal inpatients. Brain Stimul. 7, 421-431. doi: 10.1016/j.brs.2014.03.006

George, M. S., Wassermann, E. M., Kimbrell, T. A., Little, J. T., Williams, W. E., Danielson, A. L., et al. (1997). Mood improvement following daily left prefrontal repetitive transcranial magnetic stimulation in patients with depression: a placebo-controlled crossover trial. Am. J. Psychiatry 154, 1752-1756. doi: 10.1176/ajp.154.12.1752

Goldental, A. (2014). A computational paradigm for dynamic logic-gates in neuronal activity. Front. Comput. Neurosci. 8:52. doi: 10.3389/fncom. 2014.00052

Goldental, A., Sabo, P., Sardi, S., Vardi, R., and Kanter, I. (2016). Mimicking collective firing patterns of hundreds of connected neurons using a singleneuron experiment. Front. Neurosci. 9:508. doi: 10.3389/fnins.2015.00508

Goldental, A., Vardi, R., Sardi, S., Sabo, P., and Kanter, I. (2015). Broadband macroscopic cortical oscillations emerge from intrinsic neuronal response failures. Front. Neural Circuits 9:65. doi: 10.3389/fncir.2015.00065

Hamada, M., Terao, Y., Hanajima, R., Shirota, Y., Nakatani-Enomoto, S., Furubayashi, T., et al. (2008). Bidirectional long-term motor cortical plasticity and metaplasticity induced by quadripulse transcranial magnetic stimulation: quadripulse stimulation of the human motor cortex. J. Physiol. 586, 3927-3947. doi: 10.1113/jphysiol.2008.152793

Hausmann, A., Kemmler, G., Walpoth, M., Mechtcheriakov, S., KramerReinstadler, K., Lechner, T., et al. (2004). No benefit derived from repetitive transcranial magnetic stimulation in depression: a prospective, single centre, randomised, double blind, sham controlled "add on" trial. J. Neurol. Neurosurg. Psychiatr. 5, 320-322. doi: 10.1136/jnnp.2002.009209

Herbsman, T., Avery, D., Ramsey, D., Holtzheimer, P., Wadjik, C., Hardaway, F., et al. (2009). More lateral and anterior prefrontal coil location is associated with better repetitive transcranial magnetic stimulation antidepressant response. Biol. Psychiatry 66, 509-515. doi: 10.1016/j.biopsych.2009.04.034

Herwig, U., Fallgatter, A. J., Höppner, J., Eschweiler, G. W., Kron, M., Hajak, G., et al. (2007). Antidepressant effects of augmentative transcranial magnetic stimulation. Br. J. Psychiatry 191, 441-448. doi: 10.1192/bjp.bp.106. 034371

Hirayama, A., Saitoh, Y., Kishima, H., Shimokawa, T., Oshino, S., Hirata, M., et al. (2006). Reduction of intractable deafferentation pain by navigation-guided repetitive transcranial magnetic stimulation of the primary motor cortex. Pain 122, 22-27. doi: 10.1016/j.pain.2005.12.001

Hosomi, K., Shimokawa, T., Ikoma, K., Nakamura, Y., Sugiyama, K., Ugawa, Y., et al. (2013). Daily repetitive transcranial magnetic stimulation of primary motor cortex for neuropathic pain: a randomized, multicenter, double-blind, crossover, sham-controlled trial. Pain 154, 1065-1072. doi: 10.1016/j.pain.2013.03.016

Huang, Y.-Z., Edwards, M. J., Rounis, E., Bhatia, K. P., and Rothwell, J. C. (2005). Theta burst stimulation of the human motor cortex. Neuron 45, 201-206. doi: $10.1016 /$ j.neuron.2004.12.033
Huang, Y.-Z., Rothwell, J. C., Chen, R.-S., Lu, C.-S., and Chuang, W.-L. (2011). The theoretical model of theta burst form of repetitive transcranial magnetic stimulation. Clin. Neurophysiol. 122, 1011-1018. doi: $10.1016 /$ j.clinph.2010.08.016

Irlbacher, K., Kuhnert, J., Röricht, S., Meyer, B. U., and Brandt, S. A. (2006). Zentrale und periphere Deafferenzierungsschmerzen: Therapie mit der repetitiven transkraniellen Magnetstimulation? Nervenarzt 77, 1196-1203. doi: 10.1007/s00115-006-2148-1

Jetté, F., Côté, I., Meziane, H. B., and Mercier, C. (2013). Effect of single-session repetitive transcranial magnetic stimulation applied over the hand versus leg motor area on pain after spinal cord injury. Neurorehabil. Neural. Repair 27, 636-643. doi: 10.1177/1545968313484810

Jorge, R. E., Moser, D. J., Acion, L., and Robinson, R. G. (2008). Treatment of vascular depression using repetitive transcranial magnetic stimulation. Arch. Gen. Psychiatry 65, 268-276. doi: 10.1001/archgenpsychiatry.2007.45

Jorge, R. E., Robinson, R. G., Tateno, A., Narushima, K., Acion, L., Moser, D., et al. (2004). Repetitive transcranial magnetic stimulation as treatment of poststroke depression: a preliminary study. Biol. Psychiatry 55, 398-405. doi: 10.1016/j.biopsych.2003.08.017

Kang, B. S., Shin, H. I., and Bang, M. S. (2009). Effect of repetitive transcranial magnetic stimulation over the hand motor cortical area on central pain after spinal cord injury. Arch. Phys. Med. Rehabil. 90, 1766-1771. doi: 10.1016/j.apmr.2009.04.008

Khedr, E. M. (2005). Longlasting antalgic effects of daily sessions of repetitive transcranial magnetic stimulation in central and peripheral neuropathic pain. J. Neurol. Neurosurg. Psychiatry 76, 833-838. doi: 10.1136/jnnp.2004.055806

Koerselman, F., Laman, D. M., van Duijn, H., van Duijn, M. A., and Willems, M. A. (2004). A 3-month, follow-up, randomized, placebo-controlled study of repetitive transcranial magnetic stimulation in depression. J. Clin. Psychiatry 65, 1323-1328. doi: 10.4088/JCP.v65n1005

Krishnan, V., and Nestler, E. J. (2008). The molecular neurobiology of depression. Nature 455, 894-902. doi: 10.1038/nature07455

Lefaucheur, J.-P. (2004). Neurogenic pain relief by repetitive transcranial magnetic cortical stimulation depends on the origin and the site of pain. J. Neurol. Neurosurg. Psychiatry 75, 612-616. doi: 10.1136/jnnp.2003.022236

Lefaucheur, J.-P., André-Obadia, N., Antal, A., Ayache, S. S., Baeken, C., Benninger, D. H., et al. (2014). Evidence-based guidelines on the therapeutic use of repetitive transcranial magnetic stimulation (rTMS). Clin. Neurophysiol. 125, 2150-2206. doi: 10.1016/j.clinph.2014.05.021

Lefaucheur, J.-P., Drouot, X., Keravel, Y., and Nguyen, J.-P. (2001a). Pain relief induced by repetitive transcranial magnetic stimulation of precentral cortex. Neuroreport 12, 2963-2965. doi: 10.1097/00001756-200109170-00041

Lefaucheur, J. P., Drouot, X., Ménard-Lefaucheur, I., Keravel, Y., and Nguyen, J. P. (2006). Motor cortex rTMS restores defective intracortical inhibition in chronic neuropathic pain. Neurology 67, 1568-1574. doi: 10.1212/01.wnl.0000242731.10074.3c

Lefaucheur, J.-P., Drouot, X., Ménard-Lefaucheur, I., Keravel, Y., and Nguyen, J.-P. (2008). Motor cortex rTMS in chronic neuropathic pain: pain relief is associated with thermal sensory perception improvement. J. Neurol. Neurosurg. Psychiatry 79, 1044-1049. doi: 10.1136/jnnp.2007.135327

Lefaucheur, J. P., Drouot, X., and Nguyen, J. P. (2001b). Interventional neurophysiology for pain control: duration of pain relief following repetitive transcranial magnetic stimulation of the motor cortex. Neurophysiol. Clin. Neurophysiol. 31, 247-252. doi: 10.1016/S0987-7053(01)00260-X

Lefaucheur, J.-P., Ménard-Lefaucheur, I., Goujon, C., Keravel, Y., and Nguyen, J.-P. (2011). Predictive value of rTMS in the identification of responders to epidural motor cortex stimulation therapy for pain. J. Pain 12, 1102-1111. doi: 10.1016/j.jpain.2011.05.004

Lisanby, S. H., Husain, M. M., Rosenquist, P. B., Maixner, D., Gutierrez, R., Krystal, A., et al. (2009). Daily left prefrontal repetitive transcranial magnetic stimulation in the acute treatment of major depression: clinical predictors of outcome in a multisite, randomized controlled clinical trial. Neuropsychopharmacology 34, 522-534. doi: 10.1038/npp.2008.118

Loo, C. K., Mitchell, P. B., McFarquhar, T. F., Malhi, G. S., and Sachdev, P. S. (2007). A sham-controlled trial of the efficacy and safety of twice-daily rTMS in major depression. Psychol. Med. 37, 341-349. doi: 10.1017/S00332917060 09597 
Loo, C., Mitchell, P., Sachdev, P., McDarmont, B., Parker, G., and Gandevia, S. (1999). Double-blind controlled investigation of transcranial magnetic stimulation for the treatment of resistant major depression. Am. J. Psychiatry 156, 946-948. doi: 10.1176/ajp.156.6.946

Manes, F., Jorge, R., Morcuende, M., Yamada, T., Paradiso, S., and Robinson, R. G. (2001). A controlled study of repetitive transcranial magnetic stimulation as a treatment of depression in the elderly. Int. Psychogeriatr. 13, 225-231. doi: 10.1017/S1041610201007608

Mogg, A., Pluck, G., Eranti, S. V., Landau, S., Purvis, R., Brown, R. G., et al. (2008). A randomized controlled trial with 4-month follow-up of adjunctive repetitive transcranial magnetic stimulation of the left prefrontal cortex for depression. Psychol. Med. 38, 323-333. doi: 10.1017/S00332917070 01663

Monte-Silva, K., Kuo, M.-F., Hessenthaler, S., Fresnoza, S., Liebetanz, D., Paulus, W., et al. (2013). Induction of late LTP-like plasticity in the human motor cortex by repeated non-invasive brain stimulation. Brain Stimul. 6, 424-432. doi: 10.1016/j.brs.2012.04.011

Mosimann, U. P., Schmitt, W., Greenberg, B. D., Kosel, M., Müri, R. M., Berkhoff, M., et al. (2004). Repetitive transcranial magnetic stimulation: a putative addon treatment for major depression in elderly patients. Psychiatry Res. 126, 123-133. doi: 10.1016/j.psychres.2003.10.006

Nahas, Z., Kozel, F. A., Li, X., Anderson, B., and George, M. S. (2003). Left prefrontal transcranial magnetic stimulation (TMS) treatment of depression in bipolar affective disorder: a pilot study of acute safety and efficacy. Bipolar Disord 5, 40-47. doi: 10.1034/j.1399-5618.2003.00011.x

O’Reardon, J. P., Solvason, H. B., Janicak, P. G., Sampson, S., Isenberg, K. E., Nahas, Z., et al. (2007). Efficacy and safety of transcranial magnetic stimulation in the acute treatment of major depression: a multisite randomized controlled trial. Biol. Psychiatry 62, 1208-1216. doi: 10.1016/j.biopsych.2007.01.018

Padberg, F., Zwanzger, P., Keck, M. E., Kathmann, N., Mikhaiel, P., Ella, R., et al. (2002). Repetitive transcranial magnetic stimulation (rTMS) in major depression: relation between efficacy and stimulation intensity. Neuropsychopharmacology 27, 638-645. doi: 10.1016/S0893-133X(02)00338-X

Padberg, F., Zwanzger, P., Thoma, H., Kathmann, N., Haag, C., Greenberg, B. D., et al. (1999). Repetitive transcranial magnetic stimulation (rTMS) in pharmacotherapy-refractory major depression: comparative study of fast, slow and sham rTMS. Psychiatry Res. 88, 163-171. doi: 10.1016/S0165-1781(99)00092-X

Paillère Martinot, M.-L., Galinowski, A., Ringuenet, D., Gallarda, T., Lefaucheur, J.-P., Bellivier, F., et al. (2010). Influence of prefrontal target region on the efficacy of repetitive transcranial magnetic stimulation in patients with medication-resistant depression: a [18F]fluorodeoxyglucose PET and MRI study. Int. J. Neuropsychopharmacol. 13, 45-59. doi: 10.1017/S146114570900008X

Pascual-Leone, A., Rubio, B., Pallardó, F., and Catalá, M. D. (1996). Rapid-rate transcranial magnetic stimulation of left dorsolateral prefrontal cortex in drugresistant depression. Lancet 348, 233-237. doi: 10.1016/S0140-6736(96)01219-6

Pitkänen, M., Kallioniemi, E., and Julkunen, P. (2017). Effect of intertrain interval on the induction of repetition suppression of motor-evoked potentials using transcranial magnetic stimulation. PLOS ONE 12:e181663. doi: 10.1371/journal.pone.0181663

Ray, S., Nizamie, S. H., Akhtar, S., Praharaj, S. K., Mishra, B. R., and Zia-ul-Haq, M. (2011). Efficacy of adjunctive high frequency repetitive transcranial magnetic stimulation of left prefrontal cortex in depression: A randomized sham controlled study. J. Affect. Disord. 128, 153-159. doi: 10.1016/j.jad.2010.06.027

Rossi, S., Hallett, M., Rossini, P. M., and Pascual-Leone, A. (2009). Safety, ethical considerations, and application guidelines for the use of transcranial magnetic stimulation in clinical practice and research. Clin. Neurophysiol. 120, 2008-2039. doi: 10.1016/j.clinph.2009.08.016

Rossini, D., Lucca, A., Zanardi, R., Magri, L., and Smeraldi, E. (2005a). Transcranial magnetic stimulation in treatment-resistant depressed patients: a double-blind, placebo-controlled trial. Psychiatry Res. 137, 1-10. doi: 10.1016/j.psychres.2005.06.008

Rossini, D., Magri, L., Lucca, A., Giordani, S., Smeraldi, E., and Zanardi, R. (2005b). Does rTMS hasten the response to escitalopram, sertraline, or venlafaxine in patients with major depressive disorder? A double-blind, randomized, shamcontrolled trial. J. Clin. Psychiatry 66, 1569-1575. doi: 10.4088/JCP.v66n1212

Rothkegel, H., Sommer, M., and Paulus, W. (2010). Breaks during 5Hz rTMS are essential for facilitatory after effects. Clin. Neurophysiol. 121, 426-430. doi: 10.1016/j.clinph.2009.11.016

Rumi, D. O., Gattaz, W. F., Rigonatti, S. P., Rosa, M. A., Fregni, F., Rosa, M. O., et al. (2005). Transcranial magnetic stimulation accelerates the antidepressant effect of amitriptyline in severe depression: A double-blind placebocontrolled study. Biol. Psychiatry 57, 162-166. doi: 10.1016/j.biopsych.2004. 10.029

Saitoh, Y., Hirayama, A., Kishima, H., Shimokawa, T., Oshino, S., Hirata, M., et al. (2007). Reduction of intractable deafferentation pain due to spinal cord or peripheral lesion by high-frequency repetitive transcranial magnetic stimulation of the primary motor cortex. J. Neurosurg. 107, 555-559. doi: 10.3171/JNS-07/09/0555

Sardi, S., Goldental, A., Amir, H., Vardi, R., and Kanter, I. (2016). Vitality of neural networks under reoccurring catastrophic failures. Sci. Rep. 6:31674. doi: $10.1038 /$ srep31674

Sardi, R., Vardi, A., Sheinin, A., Goldental, A., and Kanter, I. (2017). New types of experiments reveal that a neuron functions as multiple independent threshold units. Sci. Rep. 7:18036. doi: 10.1038/s41598-017-18363-1

Sardi, R., Vardi, A., Goldental, A., Sheinin, A., Uzan, H., and Kanter, I. (2018) Adaptive nodes enrich nonlinear cooperative learning beyond traditional adaptation by links. Sci. Rep. 8:5100. doi: 10.1038/s41598-018-23471-7

Shirota, Y., Sommer, M., and Paulus, W. (2016). Strength-duration relationship in paired-pulse transcranial magnetic stimulation (TMS) and its implications for repetitive TMS. Brain Stimul. 9, 755-761. doi: 10.1016/j.brs.2016.04.019

Simons, L. E., Elman, I., and Borsook, D. (2014). Psychological processing in chronic pain: a neural systems approach. Neurosci. Biobehav. Rev. 39, 61-78. doi: 10.1016/j.neubiorev.2013.12.006

Sommer, M., Tergau, F., Wischer, S., and Paulus, W. (2001). Paired-pulse repetitive transcranial magnetic stimulation of the human motor cortex. Exp. Brain Res. 139, 465-472. doi: 10.1007/s002210100791

Su, T.-P., Huang, C.-C., and Wei, I.-H. (2005). Add-on rTMS for medicationresistant depression: a randomized, double-blind, sham-controlled trial in chinese patients. J. Clin. Psychiatry 66, 930-937. doi: 10.4088/JCP.v66 n0718

Triggs, W. J., Ricciuti, N., Ward, H. E., Cheng, J., Bowers, D., Goodman, W. K., et al. (2010). Right and left dorsolateral pre-frontal rTMS treatment of refractory depression: a randomized, sham-controlled trial. Psychiatry Res. 178, 467-474. doi: 10.1016/j.psychres.2010. 05.009

Vardi, R., Goldental, A., Marmari, H., Brama, H., Stern, E. A., Sardi, S., et al. (2015). Neuronal response impedance mechanism implementing cooperative networks with low firing rates and $\mu$ s precision. Front. Neural. Circuits 9:29. doi: 10.3389/fncir.2015.00029

Vardi, R., Timor, R., Marom, S., Abeles, M., and Kanter, I. (2012). Synchronization with mismatched synaptic delays: A unique role of elastic neuronal latency. EPL Europhys. Lett. 100:48003. doi: 10.1209/0295-5075/100/48003

Wilson, M. T., Fung, P. K., Robinson, P. A., Shemmell, J., and Reynolds, J. N. (2016). Calcium dependent plasticity applied to repetitive transcranial magnetic stimulation with a neural field model. J. Comput. Neurosci. 41, 107-125. doi: 10.1007/s10827-016-0607-7

Conflict of Interest Statement: The authors declare that the research was conducted in the absence of any commercial or financial relationships that could be construed as a potential conflict of interest.

Copyright (c) 2018 Halawa, Goldental, Shirota, Kanter and Paulus. This is an openaccess article distributed under the terms of the Creative Commons Attribution License (CC BY). The use, distribution or reproduction in other forums is permitted, provided the original author(s) and the copyright owner are credited and that the original publication in this journal is cited, in accordance with accepted academic practice. No use, distribution or reproduction is permitted which does not comply with these terms. 